\title{
CARACTERÍSTICAS Y MANEJO BÁSICO DE LOS CERDOS DE COMPAÑÍA
}

\author{
Sebastián Dorado-Montengro ${ }^{1}$, Jesenia Vásquez-Vargas²
}

\section{RESUMEN}

En las últimas décadas, el cerdo de compañía ha ganado gran popularidad entre las personas de zonas urbanas que desean una mascota en el hogar. No obstante, para asegurar el bienestar de los animales, resulta indispensable conocer con detalle las características propias de las especies a tratar, tales como el comportamiento, morfología, alimentación, necesidades y cuidados. La presente revisión tiene como objetivo servir de guía básica de manejo de los cerdos de compañía, de modo que tanto comerciantes como dueños puedan garantizar la vida prospera de estos animales.

Palabras clave: morfología, bienestar, comportamiento, alimentación, mascotas

\section{ABSTRACT}

Characteristics and basic management of minipigs. In recent decades, minipigs have gained great popularity amongst people in urban areas who want a pet at home. However, to ensure the welfare of animals it is essential to know in detail the characteristics of the species such as, behavior, morphology, feeding, and care. The aim of this review is to serve as a basic guide for the care of minipigs, so that both sellers and owners guarantee a long and healthy life for these animals.

Key words: morphology, welfare, behavior, feeding, pets

\footnotetext{
1 Universidad de Costa Rica, Escuela de Zootecnia y Centro de Investigaciones en Nutrición Animal. San José, Costa Rica. Autor para correspondencia: sebastian.dorado@ucr.ac.cr ${ }^{2}$ Zoológico Nacional y Jardín Botánico Simón Bolivar, San José, Costa Rica. 


\section{INTRODUCCIÓN}

En la sociedad contemporánea, el cerdo de bajo porte (minipig) se ha posicionado como uno de los animales con mayor popularidad dentro de la categoría de animales de estimación. Con este auge, cada vez más adeptos, acuden a la Escuela de Zootecnia de la Universidad de Costa Rica para obtener información sobre el cuidado y manejo básico que se debe brindar a estas mascotas.

Debido a la falta de material bibliográfico de esta índole, con respaldo técnico-científico, el presente documento se perfila como una guía de apoyo con información de fácil aplicabilidad y entendimiento. A continuación, se presentan características morfológicas, así como pautas básicas de manejo sanitario y prácticas de manejo, nutrición y alimentación, para cerdos de bajo porte, mantenidos como animales de compañía.

Tal como lo dicta la Ley No. 7451 "Bienestar de los animales", de la República de Costa Rica, resulta responsabilidad de los dueños, garantizarle a los animales de compañía las condiciones vitales básicas y un manejo apropiado según las buenas prácticas de seguridad; esto para garantizar la buena salud y el bienestar de los animales.

\section{Características principales y morfológicas}

El cerdo doméstico comercial presenta como nombre científico Sus scrofa domestica. No obstante, según algunos reportes existen alrededor de 16 sub especies de Sus scrofa (McAnulty et al., 2015), y al menos 45 razas o cruces de cerdos de bajo porte alrededor del mundo (Smith y Swindle, 2006). Esta amplia gama genética ha llevado a que los minipigs presenten gran variabilidad de características raciales como tamaño, peso, color y morfología. Cabe aclarar entonces, que la palabra "minipig" no se destina a una raza específica, sino que emplea para referirse a todos los cerdos de bajo porte.

Siendo así, para lograr una clasificación de los cerdos de compañía, la Asociación Americana de Minipigs estableció la siguiente categorización de los animales (Cuadro 1), según su altura (del piso a la cruz) y edad, dejando de lado la raza/cruce o su peso. Cabe mencionar que la omisión de las características mencionadas anteriormente, permite que la población de cerdos inscritos en esta Asociación sea altamente heterogénea y no permite hacer una evaluación más rigurosa de los animales. 
Cuadro 1. Clasificación de minipigs por altura y edad.

\begin{tabular}{cc}
\hline Categoría & Descripción \\
\hline 1 & Máximo 14 pulgadas, equivalente a 35,5 centímetros \\
2 & Entre 14 y 16 pulgadas (35,5 a 40,6 centímetros) \\
3 & Entre 16 y 18 pulgadas (40,6 a 45,7 centímetros) \\
4 & Entre 18 y 20 pulgadas (45,7 a 50,8 centímetros) \\
J & Juvenil, antes de los 16 meses de edad \\
A & Adolescente, de los 16 a los 5 años de edad \\
M & Adulto, después de los 5 años de edad \\
\hline
\end{tabular}

Fuente: AMPA American Mini Pig Association (s.f.).

A modo de ejemplo, al comparar un Göttingen, un cerdo vietnamita y un borrego (cerdo criollo costarricense), se puede observar las grandes diferencias que existen entre algunas razas de minipigs. Mientras el Göttingen presenta un peso promedio adulto (alrededor de 2 a 3 años de edad) de entre 30 a $35 \mathrm{~kg}$ y una altura de 25 a $35 \mathrm{~cm}$, el cerdo vietnamita, a la misma edad, puede exhibir un peso de entre 75 y $100 \mathrm{~kg}$ y una altura de 40 a $60 \mathrm{~cm}$. En el caso del borrego, con la misma edad, se puede presentar un peso de entre 45 y $60 \mathrm{~kg}$, con un altura de entre 50 y $65 \mathrm{~cm}$.

Como características reproductivas y de longevidad, se estima que todos los cerdos de compañía consiguen su madurez sexual entre los 3 y los 7 meses de edad, siendo los machos más precoces que las hembras (Bode et al., 2010; Peter et al., 2016); el estro (celo) tarda de 30 a 72 horas, el período gestacional es de 114 días y tienen un promedio de vida entre los 15 y 20 años, variando según las razas (Zeltner, 2013).

\section{Comportamiento}

Los cerdos en general son animales sociales y jerárquicos. En la naturaleza, acostumbran vivir en grupos con hembras reproductoras y sus camadas, mientras que los machos presentan un estilo de vida más solitario una vez que se vuelven sexualmente activos (Zeltner, 2013). 
Estos animales muestran gran inteligencia y capacidad cognitiva, como resolución de problemas y retención de información (memoria), (Held et al., 2002; Held et al., 2005; Kouwenberg et al., 2009). Aún así, existen diferencias comportamentales según la raza, el sexo y la edad (Salaun y Val-Laillet, 2013).

Para cualquier cerdo de estimación, el uso de distractores y la realización de actividades físicas contribuyen para evitar la presencia de problemas por ansiedad (Puppe et al., 2007), como las esterotipias (comportamientos repetitivos generados por estrés). Para ello, se debe ser ingenioso en el uso y construcción de juguetes y se debe considerar cambiarlos con cierta periodicidad para que no pierdan el efecto distractor al acostumbrarse el animal a ellos. Algunos ejemplos son: cadenas pendulantes para morder, trozos de frutas en cadenas pendulantes, bloques de hielo o frutas congeladas, sábanas, cobijas o cajas de cartón.

Siempre que se coloque algún tipo de juguete se recomienda supervisar la interacción del animal con el objeto, a fin de evitar lesiones o posibles atragantamientos. Es responsabilidad del dueño cerciorarse que el juguete no represente un peligro para el animal en caso de que se quiebre.

La curiosidad es otra cualidad que caracteriza a los cerdos. A pesas de comportarse tímidamente con los objetos y personas nuevas, ellos acostumbran oler y probar los objetos que le llaman la atención (Ellegard et al., 2010) como método de reconocimiento. La mayor problemática con esta conducta, es que puede llevarlos a morder y romper objetos si se desenvuelven dentro de las casas, por lo que es importante entrenarlos desde pequeños.

Para esto, varias metodologías de entrenamiento han sido desarrolladas basándose principalmente en el uso de refuerzos positivos (premios o caricias) (McGlone et al., 2001). Al ser animales muy inteligentes y con buena memoria, les resulta con facilidad aprender gran cantidad de comandos y reglas de convivencia. No obstante, al tener la capacidad de recordar tanto las buenas como las malas experiencias, bajo un manejo inadecuado, los cerdos de compañía también pueden presentar miedos por traumas que desencadenan en comportamientos poco deseables en mascotas familiares.

Otro factor que se debe considerar al momento de adquirir un cerdo de estimación, es el hecho de cómo se debe abordar el establecimiento de jerarquía y como responder ante 
eventos de agresión por parte del animal. En una investigación donde se trabajó con 222 dueños/criadores de cerdos vietnamitas, se observó que el 64\% de los entrevistados reportó que sus cerdos han presentado gestos agresivos al menos una vez (mordiscos al aire, empujado o mordidas efectivas), mientras que el $31 \%$ aseguró que sus animales presentaban actos de agresión frecuente, al menos una vez al mes (Tynes et al., 2007).

Tradicionalmente, la esterilización tanto de machos como de hembras, ha sido recomendada como método para apaciguar la agresividad. No obstante, en el estudio anteriormente mencionado, no se observaron diferencias comportamentales significativas entre machos castrados, hembras enteras y hembras castradas (no se trabajó con machos enteros). Únicamente observaron una relación con la disminución en los eventos de agresividad cuando había la presencia de otro cerdo.

A pesar de existir la teoría de la predisposición congénita a la agresividad, no hay manera para predecir de forma confiable, si un cerdo será agresivo o no con solo la observación de un individuo a temprana edad (Ardiaca, 2008). De este modo, la educación y el método de corrección resultan de gran relevancia para prevenir y tratar el problema.

El comportamiento agresivo del animal puede agravarse según la respuesta que reciba por parte del humano, la cual debe ser puntual y categórica sin recurrir al maltrato. El huir, dejar caer las cosas o hasta entregar la comida como consecuencia del ataque, puede ser considerado por el animal como un refuerzo positivo (Tynes, 2001). Un empujón fuerte en la escápula, con una vocalización acentuada ¡NO! puede detonar que el animal se aleje como acto de sumisión; en ese momento perseguirlo o espantarlo un poco podría simular la acción de un cerdo dominante. En el caso de agresiones por parte de cerdos pesados, el uso de una tabla de manejo para sujetarlos e inmovilizarlos contra una pared $u$ otra superficie, es altamente recomendable (Tynes, 2001; Ardiaca, 2008). El establecimiento de una superioridad jerárquica por parte del dueño sobre el cerdo, definirá en gran medida el comportamiento agresivo del animal.

\section{Higiene y cuidados}

Al igual que los cerdos comerciales, los minipigs requieren de condiciones ambientales muy específicas para manejarse dentro de su zona de confort. Para la raza Göttingen, animales recién nacidos necesitan estar en un ambiente con temperaturas de entre $30^{\circ} \mathrm{C}$ 
y $32^{\circ} \mathrm{C}$, disminuyendo a $26^{\circ} \mathrm{C}$ a la cuarta semana de edad; ya para la semana $34-36$ de edad, la temperatura puede rondar los $17^{\circ} \mathrm{C}$ (Ellegaard et al., 2010). Se debe recordar que los animales de esta raza presentan baja presencia de grasa corporal en relación con otros cerdos de compañía, por lo que se podría pensar que animales más grasosos de otras razas, tienen mayor capacidad para resistir bajas temperaturas.

En relación al manejo de los lechones, el Apéndice A del Convenio Europeo para la protección de animales vertebrados utilizados para experimentos y otros fines científicos (ETS 123) recomienda que las crías de los cerdos minipigs no se deben destetar antes de las 4 semanas de edad, con el objetivo de disminuir el estrés que esta práctica pueda generar. A nivel comercial se debe procurar destetar los cerditos como mínimo a los 2 meses de edad, procurando una mayor sobrevivencia y bienestar post destete y al momento de su venta.

A continuación se presentan algunas recomendaciones a considerar sobre cuidados y manejo sanitario de los cerdos de estimación:

- A diferencia de lo que muchas personas piensan, los cerdos son animales muy aseados. Instintivamente, tienen la capacidad de establecer y destinar áreas separadas para comer, defecar y orinar. Por consiguiente, en el recinto en que se desarrollen, el dueño debe proveer un área de comedero y un área donde el animal pueda hacer sus deposiciones.

- Su piel requiere de mucha hidratación, y si son de colores claros, pueden sufrir de quemaduras solares. Por esto, se debe asegurar que en las instalaciones exista un área con sombra y/o que pueda cubrirse de la lluvia, si el cerdo así lo desea. Igualmente, si el cerdo no tiene acceso a lodo o a alguna charca con agua, hay que considerar aplicarles una vez por semana vaselina sin fragancia, crema neutra para bebé o en su defecto bloqueador solar para infantes.

- Además, se recomienda dar un baño al animal cada 7-15 días. Este momento resulta aprovechable para revisar tanto la condición/aspecto de la piel como de las pezuñas.

- Existen muchas experiencias positivas en cuanto a la utilización de cajas de arena como áreas sanitarias. Con el entrenamiento adecuado, libre de estrés y maltrato, 
los minipigs consiguen aprender a utilizar estas cajas, siendo una alternativa interesante cuando se desarrollan en casas de habitación; de igual modo, deben colocarse en áreas separadas a las áreas de descanso y de los comederos, y deben limpiarse diariamente.

- En relación al control sanitario, se recomienda realizar chequeos veterinarios trimestrales en animales menores a un año de edad; para animales mayores las visitas pueden realizarse cada 6 meses. Como parte del chequeo general, se debe llevar el control de las desparasitaciones (preferiblemente si responden a exámenes coprológicos previos), peso, crecimiento, estado de las pezuñas, colmillos y piel. Si no hay interés de reproducir los animales, resulta importante consultar sobre la posibilidad de castrarlos, sin importar el sexo.

- No se recomienda tener cerdos de mascotas en lugares cercanos a granjas comerciales de cerdos. Debido a las presentaciones en las que se comercializan las vacunas, resulta inviable o muy costoso vacunar a los minipigs; esto podría tener repercusiones negativas relacionadas a problemas de salud pública.

\section{Alimentación}

La nutrición y las prácticas de alimentación resultan aspectos determinantes en la buena salud y longevidad de los minipigs. Estos animales presentan la tendencia a sufrir de obesidad, y por ello si se descuida la alimentación que se ofrece, rápidamente subirán de peso.

El hecho de que los cerdos sean omnívoros (comen tanto productos de origen animal como vegetal) no significa que se pueden alimentar con la misma dieta que los humanos consumen. Cada especie presenta requerimientos nutricionales específicos que pueden variar según el estado fisiológico y la edad del individuo. En el caso de los cerdos de bajo porte, la información disponible sobre requerimientos nutricionales resulta muy escasa, no obstante igualmente hay consideraciones básicas que se deben respetar:

- $\quad$ Disponibilidad de agua potable para su consumo, en todo momento.

- Se pueden utilizar ciertos alimentos para consumo humanos (alimentación fresca) o alimentos balanceados específicos para cerdos. 
- Para alimentación fresca, se recomienda ofrecer verduras de hoja verde como lechuga, mostaza china y espinacas, entre otros. También se pueden usar frutas preferiblemente con bajos niveles de azúcar y acidez (en relación 2:1 verduras: frutas), granos, semillas y algunos productos de origen animal que sean bajos en grasa. Valorar dar el doble de verduras que de frutas. Al ofrecer este tipo de alimentación se debe balancear la dieta, por lo que es altamente recomendable procurar un nutricionista animal (Zootecnista). Esto evitará generar desordenes nutricionales en el animal.

- Se puede ofrecer alimento específico para cerdo acompañado de algún ingrediente fibroso como salvadillo de trigo, afrecho de arroz o harina de coquito de palma africana:

- Alimento de inicio: Ofrecer los primeros 5-6 meses de edad.

- Alimento engorde NO medicado y sin ractopamina: Ofrecer de los 56 meses al año de edad.

- Alimento de gestación: después del año de edad. Este es un alimento alto en fibra por lo que ayuda a controlar el peso.

- También puede considerarse la suplementación con compuestos vitamínicos, de Zinc, de Selenio, cápsulas de aceite de coco o pescado, extracto de Yucca Schidigera, probioticos, prebióticos, entre otros; siguiendo las recomendaciones de un nutricionista animal. Estos productos se pueden adquirir en macrobióticas y las dosis de uso dependerán de la composición de cada uno.

- Sin importar si se utilizan alimentos frescos o alimentos balanceados, se recomienda ofrecerlos proporcionalmente según el peso del animal. Es decir, se recomienda dar la cantidad equivalente de $2,5 \%$ a $1,5 \%$ del peso vivo dependiendo de la condición corporal y estado fisiológico. En relación al sexo, se considera normal que las hembras consuman menos alimento que los machos.

- Si se respetan las cantidades de alimento recomendadas, rara vez los cerdos dejarán comida después de pocos minutos de haberse ofrecido. Sin embargo, si se presenta rechazo por los alimentos, es recomendable retirar los sobrantes del 
plato o comedero, 30 minutos después de ofrecidos (sobre todo si son alimentos frescos por posibles problemas de rancidez).

- Los alimentos deben ofrecerse 1 o 2 veces al día. Según la empresa Ellegaard Göttingen Minipigs ${ }^{\circledR}$, dedicada a la reproducción y venta de cerdos de la raza Göttingen, antes de los 6-7 meses se recomienda ofrecer la comida dividida en dos tiempos de alimentación; después de esa edad, se puede ofrecer una vez al día.

No es recomendable alimentar con: Dulces, alimentos de panadería, alimentos procesados ni excesivamente salados, alimentos balanceados para carnívoros domésticos (perros y gatos) ni alimentos con exceso de grasa. La alimentación con uso exclusivo de alimento para conejo o caballo, puede conllevar en el tiempo a problemas metabólicos y de salud, además en el mercado existen alimentos balanceados con grandes diferencias en su composición nutricional.

En la naturaleza, los cerdos usualmente buscan la comida escarbando con el hocico en la tierra. Se puede promover este tipo de comportamiento poniendo la comida esparcida en diferentes puntos a lo largo del patio o recinto; como beneficio adicional, al obligar al animal a buscar el alimento se promueve una mayor actividad física.

A continuación, a modo de ejemplo se presentan dietas balanceadas para tres categorías de cerditos raza Göttingen (Cuadros 2, 3 y 4). La edad de pubertad fue considerada como parámetro para el cambio de alimentación, presentándose alrededor de los 6 meses de edad. Asimismo, se considera un cerdo adulto después de 2 años de edad, donde en el caso de esta raza, se puede alcanzar el tamaño máximo del animal adulto. Es en este momento en que la curva de crecimiento comienza a estabilizarse y se torna de gran importancia el mantenimiento y control del peso corporal.

Las siguientes dietas representan la cantidad de alimento total a ofrecer en un día por animal. En caso de se quiera ofrecer el alimento en más de un tiempo de comida, se debe dividir la cantidad presentada entre el número de ofertas que se desean realizar. Además, cabe resaltar que la cantidad de alimento reportada en las primeras dos fases, representa el máximo consumo recomendado por día al momento del cambio de alimentación. 
Cuadro 2. Propuesta de dietas para la etapa de inicio para cerdos de compañía (de los 23 meses y hasta los 6 meses de edad), considerando un consumo diario de $375 \mathrm{~g}$ (peso $15 \mathrm{~kg}$ ).

\begin{tabular}{ccc}
\hline Tipo de dieta & Ingrediente & Cantidad (g) \\
\hline Exclusiva con A. balanceados & A. balanceado* & 345 \\
& Semolina & 30 \\
Fresca & & \\
& A. balanceado* & 125 \\
& Tomate & 75 \\
& Papaya & 65 \\
& Chile & 50 \\
& Sandía & 45 \\
& Manzana & 15 \\
\hline${ }^{*}$ Características del A. balanceado: 19\%Proteína Cruda, 4\% Fibra Cruda, 3450 kcal/kg EM
\end{tabular}

Cuadro 3. Propuesta de dietas para la etapa de adulto joven para cerdos de compañía (de 6 meses a 24 meses), considerando un consumo diario de $500 \mathrm{~g}$ (peso 25 $\mathrm{kg})$.

\begin{tabular}{ccc}
\hline Tipo de dieta & Ingrediente & Cantidad (g) \\
\hline Exclusiva con A. balanceados & A. balanceado* & 235 \\
& Semolina & 235 \\
Fresca & Producto fibroso** & 30 \\
& A. balanceado* & 180 \\
& Chile & 55 \\
& Sandía & 50 \\
& Tomate & 50 \\
& Producto fibroso** & 45 \\
& Papaya & 40 \\
** Producto fibroso con 35\% Fibra Cruda & Banano & 40 \\
& Manzana & 30 \\
& Afrecho & 10 \\
Nutrición Animal Tropical 13(1): 1-14. ISSN: 2215-3527/ 2019 &
\end{tabular}


Cuadro 4. Propuesta de dietas para la etapa para la etapa de adulto para cerdos de compañía (mayor a 24 meses), considerando un consumo diario de $525 \mathrm{~g}$ (peso $35 \mathrm{~kg}$ ).

\begin{tabular}{ccc}
\hline Tipo de dieta & Ingrediente & Cantidad (g) \\
\hline Exclusiva con A. balanceados & Semolina & 490 \\
& A. balanceado* & 35 \\
Fresca & A. balanceado* & 165 \\
& Producto fibroso ** & 60 \\
& Acemite & 50 \\
& Tomate & 50 \\
& Banano & 50 \\
& Sandía & 40 \\
& Chile & 40 \\
& Manzana & 40 \\
\hline
\end{tabular}

${ }^{*}$ Características del A. balanceado: 14\% Proteína Cruda, 6\% Fibra Cruda, 3350 kcal/kg EM

** Producto fibroso con 35\% Fibra Cruda

Como parte de las consideraciones que se deben tener al comprar una mascota, es de gran importancia ponderar el valor de la manutención de la misma. En el Cuadro 5 se presentan los costos aproximados de las dietas expuestas anteriormente. A estos valores, se les debe adicionar los costos por conceptos de consulta veterinaria y cuidados generales.

Cuadro 5. Estimación de costos en alimentación diaria, mensual y por duración de etapa en colones costarricenses $(\mathbb{W})$.

\begin{tabular}{ccccccc}
\hline \multirow{2}{*}{ Etapa } & \multicolumn{2}{c}{ Diario $(\mathbb{L})$} & \multicolumn{2}{c}{ Mensual $(\mathbb{L})$} & \multicolumn{2}{c}{ Por etapa $(\mathbb{L})$} \\
\cline { 2 - 7 } & Fresco & Seca & Fresco & Seca & Fresco & Seca \\
\hline Inicio* $^{*}$ & 228,8 & 114,3 & 6978,4 & 3486,2 & 27913,6 & 13944,8 \\
Juvenil $^{* *}$ & 1031,2 & 588,5 & 31451,6 & 17949,3 & 566128,8 & 323087,4 \\
Adulto*** $^{*}$ & 1206,3 & 123,8 & 36792,2 & 3775,9 & 441506,4 & 45310,8 \\
\hline
\end{tabular}

* La etapa de inicio representa un período de 4 meses.

${ }^{* *}$ La etapa juvenil representa un período de 18 meses.

*** La etapa de la alimentación del adulto representa un período de 1 año.

Tipo de cambio: US\$ compra: $\mathbb{C} 576,50$. US\$ venta: $\mathbb{L} 583.14$ 
Para la estimación de los costos de las dietas, se cotizaron los precios del alimento fibroso en 3 diferentes macrobióticas ubicadas en el área metropolitana de San José y se obtuvo un precio promedio. Además, los costos de las frutas y verduras utilizadas fueron cuantificaron según los boletines de precios del Sistema de Información de Mercados Mayoristas (SIMM) correspondientes a las fechas del 10 y 20 de setiembre, 2018.

La duración por etapa considera el período en que los animales deberían consumir la dieta propuesta, siendo en el caso de inicio 4 meses y para juveniles 18 meses; para la etapa de adulto de presenta el costo anual para efectos del ejercicio. Esta alimentación se propone como base para el mantenimiento durante la mayor parte de su vida, teniendo una expectativa de vida de 12 a 15 años. Además, conforme el animal avanza en edad (etapa geriátrica), se debe considerar el estado de salud para realizar posibles ajustes en la dieta según recomendaciones profesionales.

\section{CONSIDERACIONES FINALES}

En Costa Rica, según la ley No. 7451 del Gobierno de la República, resulta una obligación por parte de los dueños de animales de compañía, velar por el bienestar de sus mascotas rechazando cualquier indicio de maltrato animal. De este modo, es primordial conocer con detalle las necesidades de los animales, su comportamiento, la nutrición y su debido manejo.

Actualmente existen todavía lagunas de conocimiento relacionadas a los requerimientos nutricionales de los cerdos de compañía, debido a la gran heterogeneidad que existe entre las razas y cruces que los componen. Ante esto, la experiencia de campo toma gran relevancia a modo de identificar que alimentos podrían provocar reacciones alérgicas, deficiencias, enfermedades o cualquier otro efecto nocivo para la salud.

Bajo las condiciones adecuadas, los minipigs pueden desarrollarse perfectamente como animales de compañía. Su gran inteligencia les permite aprender y adaptarse a gran variedad de condiciones, logrando convivir en armonía con personas y otros animales. Asimismo, su presencia puede acarrear amplios beneficios psicológicos, sociales y de salud a sus dueños. 


\section{LITERATURA CITADA}

AMPA (American Mini Pig Association). S.f. Education: Mini Pig Size Classifications. IN: https://americanminipigassociation.com/mini-pig-education/mini-pig-sizeclassifications/

Ardiaca, M. 2008. Problemas de comportamiento en mascotas no recomendables como mascotas por sus problemas de comportamiento. Clínica Veterinaria de Pequeños Animales 28 (4): 245-250.

Bode, G., P. Clausing, F. Gervais, J. Loegsted, J. Luft, V. Nogues, y J. Sims. 2010. The utility of the minipig as an animal model in regulatory toxicology. Journal of Pharmacological and Toxicological Methods 62 (3): 196-220.

Consejo Europeo. 2006. Appendix A of the European Convention for the protección of vertébrate animals used for experimental and other scientific purposes (ETS 123). Guidelines for accommodation and care of animals (article 5 of the convention). 109p. IN: https://www.coe.int/en/web/conventions/full-list/-/conventions/treaty/123

Ellegard, L., A. Cunningham, S. Edwards, N. Grand, T. Nevalainen, M. Prescott, y T. Schuurman. 2010. Welfare of the minipig with special reference to use in regulatory toxicology studies. Journal of Pharmacological and Toxicological Methods 62 (3): 167-183.

Held, S, J. Baumgartner, A. KilBride, R.W. Byrne, y M. Mendl. 2005. Foraging behaviour in domestic pigs (Sus scrofa): remembering and prioritizing food sites of different value. Animal Cognition 8(2): 114-121.

Kouwenberg, A.L., C.J. Walsh, B.E. Morgan, y G.M. Martin. 2009 Episodic-like memory in crossbred Yucatan minipigs (Sus scrofa). Applied Animal Behaviour Science 117: 165-172

McAnulty, P.A., A.D. Dayan, N.C. Ganderup, K.L. y K.L Hastings. 2012. The minipig in biomedical research. CRC Press, Taylor \& Francis Group. Florida, United States. $347 p$.

McGlone, J.J., S.E. Curtis, y T.R. Houpt. 2001. Husbandry, anesthesia, and surgery. In: Pond WG, Mersmann HJ, eds. Biology of the Domestic Pig. Ithaca: Comstock Publishing Associates.

Peter, B., E.P.C.T. De Rijk, y A. Zeltner. 2016. Sexual Maturation in female Göttingen Minipig. Toxicologic Pathology 44(3): 482-485.

Puppe, B., K. Ernst, P.C. Schön, y G. Manteuffel. 2007. Cognitive enrichment affects behavioural reactivity in domestic pigs. Applied Animal Behaviour Science 105 (13):75-86. 
Salaun, M.C., y D. Val-Laillet. 2013. Study of animal behavior and welfare of miniature pigs in France. Livestock Research Institute, Council of Agriculture, Symposium Bilateral Symposium on Miniature Pigs for Biomedical Research in Taiwan and France. IN: https://hal.archives-ouvertes.fr/hal-01178273/document

SIMM (Sistema de Información de Mercados Mayoristas). Boletín de precios. IN: http://www.pima.go.cr/simm/

Smith A.C., y M.M. Swindle. 2006. Preparation of swine for the laboratory. ILAR Institute for Laboratory Animal Research Journal 47(4):358-363

Tynes, V.V. 2001. Behavior of miniature pet pigs. Veterinary Clinics of North America: Exotic Animal Practice 4(3): 713-734.

Tynes, V.V., B.L. Hart, y M.J. Bain. 2007. Human-directed aggression in miniature pet pigs. Journal of the American Veterinary Medical Assoccciation 230(3): 385-389.

Zeltner, A. 2013. Handling, dosing and training of the Göttingen Minipig. Ellegaard Göttingen Minipigs, Educational package. 44p. IN: http://minipigs.dk/fileadmin/filer/Education_package_New/Handling_Dosing__Tra ining.pdf 